Article

\title{
New Applications of the Bernardi Integral Operator
}

\author{
Shigeyoshi Owa ${ }^{1, *(D)}$ and H. Özlem Güney ${ }^{2}$ (D) \\ 1 “1 Decembrie 1918” University Alba Iulia, 510009 Alba-Iulia, Romania \\ 2 Department of Mathematics, Faculty of Science Dicle University, 21280 Diyarbakır, Turkey; \\ ozlemg@dicle.edu.tr \\ * Correspondence: shige21@ican.zaq.ne.jp
}

Received: 30 June 2020; Accepted: 16 July 2020; Published: 17 July 2020

Abstract: Let $A(p, n)$ be the class of $f(z)$ which are analytic $p$-valent functions in the closed unit disk $\overline{\mathbb{U}}=\{z \in \mathbb{C}:|z| \leq 1\}$. The expression $B_{-m-\lambda} f(z)$ is defined by using fractional integrals of order $\lambda$ for $f(z) \in A(p, n)$. When $m=1$ and $\lambda=0, B_{-1} f(z)$ becomes Bernardi integral operator. Using the fractional integral $B_{-m-\lambda} f(z)$, the subclass $T_{p, n}\left(\alpha_{s}, \beta, \rho ; m, \lambda\right)$ of $A(p, n)$ is introduced. In the present paper, we discuss some interesting properties for $f(z)$ concerning with the class $T_{p, n}\left(\alpha_{s}, \beta, \rho ; m, \lambda\right)$. Also, some interesting examples for our results will be considered.

Keywords: analytic $p$-valent function; Bernardi integral operator; Libera integral operator; fractional integral; gamma function; Miller-Mocanu lemma

\section{Introduction}

Let $A(p, n)$ be the class of functions $f(z)$ of the form

$$
f(z)=z^{p}+\sum_{k=p+n}^{\infty} a_{k} z^{k}, n \in \mathbb{N}=\{1,2,3, \ldots\}
$$

that are analytic $p$-valent functions in the closed unit disk $\overline{\mathbb{U}}=\{z \in \mathbb{C}:|z| \leq 1\}$. For functions $f(z) \in A(p, n)$, we consider

$$
B_{-1} f(z)=\frac{p+\gamma}{z^{\gamma}} \int_{0}^{z} t^{\gamma-1} f(t) d t=z^{p}+\sum_{k=p+n}^{\infty} \frac{p+\gamma}{k+\gamma} a_{k} z^{k}, \gamma \in \mathbb{N}
$$

If $p=1$, for $f(z) \in A(1, n)$

$$
B_{-1} f(z)=\frac{1+\gamma}{z^{\gamma}} \int_{0}^{z} t^{\gamma-1} f(t) d t=z+\sum_{k=n+1}^{\infty} \frac{1+\gamma}{k+\gamma} a_{k} z^{k}, \gamma \in \mathbb{N}
$$

is considered by Bernardi [1]. Therefore, $B_{-1} f(z)$ in (3) is said to be the Bernardi integral operator. Further, if $p=1$ and $\gamma=1$, for $f(z) \in A(1, n)$

$$
L_{-1} f(z)=\frac{2}{z} \int_{0}^{z} f(t) d t=z+\sum_{k=n+1}^{\infty} \frac{2}{k+1} a_{k} z^{k}
$$

is defined by Libera [2]. Therefore, $L_{-1} f(z)$ in (4) is called the Libera integral operator.

For $B_{-1} f(z)$ in (2), we consider

$$
B_{-2} f(z)=B_{-1}\left(B_{-1} f(z)\right)=z^{p}+\sum_{k=p+n}^{\infty}\left(\frac{p+\gamma}{k+\gamma}\right)^{2} a_{k} z^{k}
$$


and

$$
B_{-m} f(z)=B_{-1}\left(B_{-m+1} f(z)\right)=z^{p}+\sum_{k=p+n}^{\infty}\left(\frac{p+\gamma}{k+\gamma}\right)^{m} a_{k} z^{k}
$$

with $m \in \mathbb{N}$ and $B_{0} f(z)=f(z)$.

From the various definitions of fractional calculus of $f(z) \in A(p, n)$ (that is, fractional integrals and fractional derivatives) given in the literature, we would like to recall here the following definitions for fractional calculus which were used by Owa [3] and Owa and Srivastava [4].

Definition 1. The fractional integral of order $\lambda$ for $f(z) \in A(p, n)$ is defined by

$$
D_{z}^{-\lambda} f(z)=\frac{1}{\Gamma(\lambda)} \int_{0}^{z} \frac{f(t)}{(z-t)^{1-\lambda}} d t, \lambda>0
$$

where the multiplicity of $(z-t)^{\lambda-1}$ is removed by requiring $\log (z-t)$ to be real when $z-t>0$ and $\Gamma$ is the Gamma function.

With the above definitions, we know that

$$
D_{z}^{-\lambda} f(z)=\frac{\Gamma(p+1)}{\Gamma(p+1+\lambda)} z^{p+\lambda}+\sum_{k=p+n}^{\infty} \frac{\Gamma(k+1)}{\Gamma(k+1+\lambda)} a_{k} z^{k+\lambda}
$$

for $\lambda>0$ and $f(z) \in A(p, n)$. Using the fractional integral operator over $A(p, n)$, we consider

$$
B_{-\lambda} f(z)=\frac{\Gamma(p+\gamma+\lambda)}{\Gamma(p+\gamma)} z^{1-\gamma-\lambda} D_{z}^{-\lambda}\left(z^{\gamma-1} f(z)\right)=z^{p}+\sum_{k=p+n}^{\infty} \frac{\Gamma(k+\gamma) \Gamma(p+\gamma+\lambda)}{\Gamma(p+\gamma) \Gamma(k+\gamma+\lambda)} a_{k} z^{k},
$$

where $0 \leq \lambda \leq 1$. If $\lambda=0$ in (9), then $B_{0} f(z)=f(z)$ and if $\lambda=1$ in (9), then we see that

$$
B_{-1} f(z)=\frac{p+\gamma}{z^{\gamma}} \int_{0}^{z} t^{\gamma-1} f(t) d t
$$

With the operator $B_{-\lambda} f(z)$ given by (9), we know

$$
B_{-m-\lambda} f(z)=B_{-m}\left(B_{-\lambda} f(z)\right)=z^{p}+\sum_{k=p+n}^{\infty}\left(\frac{p+\gamma}{k+\gamma}\right)^{m} \frac{\Gamma(k+\gamma) \Gamma(p+\gamma+\lambda)}{\Gamma(p+\gamma) \Gamma(k+\gamma+\lambda)} a_{k} z^{k}
$$

where $0 \leq \lambda \leq 1$ and $m \in \mathbb{N}$. The operator $B_{-m-\lambda} f(z)$ is a generalization of the Bernardi integral operator $B_{-1} f(z)$. From the definition of $B_{-m-\lambda} f(z)$, we know that

$$
B_{-m-\lambda} f(z)=B_{-m}\left(B_{-\lambda} f(z)\right)=B_{-\lambda}\left(B_{-m} f(z)\right) .
$$

From $s$ different boundary points $z_{l}(l=1,2,3, \ldots, s)$ with $\left|z_{l}\right|=1$, we consider

$$
\alpha_{s}=\frac{1}{s} \sum_{l=1}^{s} \frac{B_{-m-\lambda} f\left(z_{l}\right)}{z_{l}^{p}}
$$

where $\alpha_{s} \in e^{i \beta} B_{-m-\lambda} f(\mathbb{U}), \alpha_{s} \neq 1,-\frac{\pi}{2} \leq \beta \leq \frac{\pi}{2}$ and $\mathbb{U}=\{z \in \mathbb{C}:|z|<1\}$ is the open unit disk. For such $\alpha_{s}$, if $f(z) \in A(p, n)$ satisfies

$$
\left|\frac{e^{i \beta \frac{B_{-m-\lambda} f(z)}{z^{p}}}-\alpha_{s}}{e^{i \beta}-\alpha_{s}}-1\right|<\rho, z \in \mathbb{U}
$$

for some real $\rho>0$, we say that the function $f(z)$ belongs to the class $T_{p, n}\left(\alpha_{s}, \beta, \rho ; m, \lambda\right)$. 
It is clear that a function $f(z) \in A(p, n)$ belongs to the class $T_{p, n}\left(\alpha_{s}, \beta, \rho ; m, \lambda\right)$ provided that the condition

$$
\left|\frac{B_{-m-\lambda} f(z)}{z^{p}}-1\right|<\rho\left|e^{i \beta}-\alpha_{s}\right|, z \in \mathbb{U},
$$

is satisfied. If we consider the function $f(z) \in A(p, n)$ given by

$$
f(z)=z^{p}+\left(\frac{p+n+\gamma}{p+\gamma}\right)^{m} \frac{\Gamma(p+\gamma) \Gamma(p+n+\gamma+\lambda)}{\Gamma(p+n+\gamma) \Gamma(p+\gamma+\lambda)} \rho\left(e^{i \beta}-\alpha_{s}\right) z^{p+n}
$$

then $f(z)$ satisfies

$$
\left|\frac{B_{-m-\lambda} f(z)}{z^{p}}-1\right|=\rho\left|e^{i \beta}-\alpha_{s}\right||z|^{m}<\rho\left|e^{i \beta}-\alpha_{s}\right| \quad, \quad z \in \mathbb{U} .
$$

Therefore, $f(z)$ given by (16) is in the class $T_{p, n}\left(\alpha_{s}, \beta, \rho ; m, \lambda\right)$.

Discussing our problems for $f(z) \in T_{p, n}\left(\alpha_{s}, \beta, \rho ; m, \lambda\right)$, we have to recall here the following lemma due to Miller and Mocanu [5,6] (refining the old one in Jack [7].)

Lemma 1. Let the function $w(z)$ given by

$$
w(z)=a_{n} z^{n}+a_{n+1} z^{n+1}+a_{n+2} z^{n+2}+\ldots, n \in \mathbb{N}
$$

be analytic in $\mathbb{U}$ with $w(0)=0$. If $|w(z)|$ attains its maximum value on the circle $|z|=r$ at a point $z_{0},\left(0<\left|z_{0}\right|<1\right)$ then there exists a real number $k \geq n$ such that

$$
\frac{z_{0} w^{\prime}\left(z_{0}\right)}{w\left(z_{0}\right)}=k
$$

and

$$
\operatorname{Re}\left(1+\frac{z_{0} w^{\prime \prime}\left(z_{0}\right)}{w^{\prime}\left(z_{0}\right)}\right) \geq k
$$

\section{Properties of Functions Concerning with the Class $T_{p, n}\left(\alpha_{s}, \beta, \rho ; m, \lambda\right)$}

We begin with a sufficient condition on a function $f(z) \in A(p, n)$ which makes it a member of $T_{p, n}\left(\alpha_{s}, \beta, \rho ; m, \lambda\right)$.

Theorem 1. If $f(z) \in A(p, n)$ satisfies

$$
\left|\frac{B_{-m-\lambda+1} f(z)}{B_{-m-\lambda} f(z)}-1\right|<\frac{\left|e^{i \beta}-\alpha_{s}\right| n \rho}{(p+\gamma)\left(1+\left|e^{i \beta}-\alpha_{s}\right| \rho\right)} \quad, z \in \mathbb{U}
$$

for some $\alpha_{s}$ given by (13) with $\alpha_{s} \neq 1$ such that $z_{g} \in \partial \mathbb{U}(g=1,2,3, \ldots, s)$, and for some real $\rho>1$, then

$$
\left|\frac{B_{-m-\lambda} f(z)}{z^{p}}-1\right|<\rho\left|e^{i \beta}-\alpha_{s}\right| \quad, z \in \mathbb{U}
$$

that is, $f(z) \in T_{p, n}\left(\alpha_{s}, \beta, \rho ; m, \lambda\right)$.

Proof. We introduce the function $w(z)$ defined by

$$
w(z)=\frac{e^{i \beta} \frac{B_{-m-\lambda} f(z)}{z^{p}}-\alpha_{s}}{e^{i \beta}-\alpha_{s}}-1=\frac{e^{i \beta}}{e^{i \beta}-\alpha_{s}}\left\{\sum_{k=p+n}^{\infty}\left(\frac{p+\gamma}{k+\gamma}\right)^{m} \frac{\Gamma(k+\gamma) \Gamma(p+\gamma+\lambda)}{\Gamma(p+\gamma) \Gamma(k+\gamma+\lambda)} a_{k} z^{k-p}\right\} .
$$


Then, $w(z)$ is analytic in $\mathbb{U}$ with $w(0)=0$ and

$$
\frac{B_{-m-\lambda} f(z)}{z^{p}}=1+\left(1-e^{-i \beta} \alpha_{s}\right) w(z)
$$

Noting that

$$
B_{-m-\lambda+1} f(z)=\frac{\gamma}{p+\gamma} B_{-m-\lambda} f(z)+\frac{1}{p+\gamma} z\left(B_{-m-\lambda} f(z)\right)^{\prime}
$$

we obtain that

$$
\frac{B_{-m-\lambda+1} f(z)}{B_{-m-\lambda} f(z)}-1=\frac{\left(1-e^{-i \beta} \alpha_{s}\right) z w^{\prime}(z)}{(p+\gamma)\left(1+\left(1-e^{-i \beta} \alpha_{s}\right) w(z)\right)}
$$

and that

$$
\left|\frac{B_{-m-\lambda+1} f(z)}{B_{-m-\lambda} f(z)}-1\right|=\frac{1}{p+\gamma}\left|\frac{\left(1-e^{-i \beta} \alpha_{s}\right) z w^{\prime}(z)}{1+\left(1-e^{-i \beta} \alpha_{s}\right) w(z)}\right|<\frac{\left|e^{i \beta}-\alpha_{s}\right| n \rho}{(p+\gamma)\left(1+\left|e^{i \beta}-\alpha_{s}\right| \rho\right)}
$$

by employing (21). Assume, to arrive at a contradiction, that there exists a point $z_{0},\left(0<\left|z_{0}\right|<1\right)$ such that

$$
\max \left\{|w(z)| ;|z| \leq\left|z_{0}\right|\right\}=\left|w\left(z_{0}\right)\right|=\rho>1
$$

Then, we can write that $w\left(z_{0}\right)=\rho e^{i \theta},(0 \leq \theta \leq 2 \pi)$ and $z_{0} w^{\prime}\left(z_{0}\right)=k w\left(z_{0}\right),(k \geq n)$ by Lemma 1 . For such a point $z_{0} \in \mathbb{U}, f(z)$ satisfies

$$
\begin{aligned}
\left|\frac{B_{-m-\lambda+1} f\left(z_{0}\right)}{B_{-m-\lambda} f\left(z_{0}\right)}-1\right| & =\frac{1}{p+\gamma}\left|\frac{\left(1-e^{-i \beta} \alpha_{s}\right) z_{0} w^{\prime}\left(z_{0}\right)}{1+\left(1-e^{-i \beta} \alpha_{s}\right) w\left(z_{0}\right)}\right| \\
& =\frac{1}{p+\gamma}\left|\frac{\left(1-e^{-i \beta} \alpha_{s}\right) k \rho}{1+\left(1-e^{-i \beta} \alpha_{s}\right) \rho e^{i \theta}}\right| \\
& \geq \frac{\left|1-e^{-i \beta} \alpha_{s}\right| n \rho}{(p+\gamma)\left(1+\left|1-e^{-i \beta} \alpha_{s}\right| \rho\right)} \\
& =\frac{\left|e^{i \beta}-\alpha_{s}\right| n \rho}{(p+\gamma)\left(1+\left|e^{i \beta}-\alpha_{s}\right| \rho\right)} .
\end{aligned}
$$

Since this contradicts our condition (21), we see that there is no $z_{0},\left(0<\left|z_{0}\right|<1\right)$ such that $\left|w\left(z_{0}\right)\right|=$ $\rho>1$. This shows us that

$$
|w(z)|=\left|\frac{e^{i \beta}\left(\frac{B_{-m-\lambda} f(z)}{z^{p}}-1\right)}{e^{i \beta}-\alpha_{s}}\right|<\rho, z \in \mathbb{U},
$$

that is, that

$$
\left|\frac{B_{-m-\lambda} f(z)}{z^{p}}-1\right|<\rho\left|e^{i \beta}-\alpha_{s}\right|, z \in \mathbb{U} .
$$

This completes the proof of the theorem.

Example 1. We consider a function $f(z) \in A(p, n)$ given by

$$
f(z)=z^{p}+a_{p+n} z^{p+n}, z \in \mathbb{U}
$$

with $0<\left|a_{p+n}\right|<\frac{1}{2 Q}$, where

$$
Q=\left(\frac{p+\gamma}{p+n+\gamma}\right)^{m} \frac{\Gamma(p+n+\gamma) \Gamma(p+\gamma+\lambda)}{\Gamma(p+\gamma) \Gamma(p+n+\gamma+\lambda)}
$$


For such $f(z)$, we have

$$
\frac{B_{-m-\lambda+1} f(z)}{B_{-m-\lambda} f(z)}=\frac{z^{p}+\left(\frac{p+n+\gamma}{p+\gamma}\right) Q a_{p+n} z^{p+n}}{z^{p}+Q a_{p+n} z^{p+n}}=\frac{1+\left(\frac{p+n+\gamma}{p+\gamma}\right) Q a_{p+n} z^{n}}{1+Q a_{p+n} z^{n}}
$$

that is, that

$$
\left|\frac{B_{-m-\lambda+1} f(z)}{B_{-m-\lambda} f(z)}-1\right|=\left|\frac{\left(\frac{n}{p+\gamma}\right) Q a_{p+n} z^{n}}{1+Q a_{p+n} z^{n}}\right|<\frac{\left(\frac{n}{p+\gamma}\right) Q\left|a_{p+n}\right|}{1-Q\left|a_{p+n}\right|} \quad, \quad z \in \mathbb{U}
$$

Now, we consider five boundary points such that

$$
\begin{aligned}
& z_{1}=e^{-i \frac{\arg \left(a_{p+n}\right)}{n}} \\
& z_{2}=e^{i \frac{\pi-6 \arg \left(a_{p+n}\right)}{n}} \\
& z_{3}=e^{i \frac{\pi-4 \arg \left(a_{p+n}\right)}{4 n}} \\
& z_{4}=e^{i \frac{\pi-3 \arg \left(a_{p+n}\right)}{3 n}}
\end{aligned}
$$

and

$$
z_{5}=e^{i \frac{\pi-2 \arg \left(a_{p+n}\right)}{2 n}}
$$

For these five boundary points, we know that

$$
\begin{gathered}
\frac{B_{-m-\lambda} f\left(z_{1}\right)}{z_{1}^{p}}=1+Q a_{p+n} e^{-\arg \left(a_{p+n}\right)}=1+Q\left|a_{p+n}\right|, \\
\frac{B_{-m-\lambda} f\left(z_{2}\right)}{z_{2}^{p}}=1+Q a_{p+n} e^{i\left(\frac{\pi}{6}-\arg \left(a_{p+n}\right)\right)}=1+\frac{\sqrt{3}+i}{2} Q\left|a_{p+n}\right|, \\
\frac{B_{-m-\lambda} f\left(z_{3}\right)}{z_{3}^{p}}=1+Q a_{p+n} e^{i\left(\frac{\pi}{4}-\arg \left(a_{p+n}\right)\right)}=1+\frac{\sqrt{2}(1+i)}{2} Q\left|a_{p+n}\right|, \\
\frac{B_{-m-\lambda} f\left(z_{4}\right)}{z_{4}^{p}}=1+Q a_{p+n} e^{i\left(\frac{\pi}{3}-\arg \left(a_{p+n}\right)\right)}=1+\frac{1+\sqrt{3} i}{2} Q\left|a_{p+n}\right|,
\end{gathered}
$$

and

$$
\frac{B_{-m-\lambda} f\left(z_{5}\right)}{z_{5}^{p}}=1+Q a_{p+n} e^{i\left(\frac{\pi}{2}-\arg \left(a_{p+n}\right)\right)}=1+i Q\left|a_{p+n}\right|
$$

Thus $\alpha_{5}$ is given by

$$
\alpha_{5}=\frac{1}{5} \sum_{l=1}^{5} \frac{B_{-m-\lambda} f\left(z_{l}\right)}{z_{l}^{p}}=1+\frac{(3+\sqrt{2}+\sqrt{3})(1+i)}{10} Q\left|a_{p+n}\right| .
$$

This gives us that

$$
\left|1-e^{-i \beta} \alpha_{5}\right|=\frac{\sqrt{2}(3+\sqrt{2}+\sqrt{3})}{10} Q\left|a_{p+n}\right|
$$

with $\beta=0$. For such $\alpha_{5}$ and $\beta$, we take $\rho>1$ with

$$
\frac{\left(\frac{n}{p+\gamma}\right) Q\left|a_{p+n}\right|}{1-Q\left|a_{p+n}\right|} \leq \frac{\left|e^{i \beta}-\alpha_{5}\right| n \rho}{(p+\gamma)\left(1+\left|e^{i \beta}-\alpha_{5}\right| \rho\right)}
$$


It follows from the above that

$$
\rho \geq \frac{10}{\sqrt{2}(3+\sqrt{2}+\sqrt{3})\left(1-2 Q\left|a_{p+n}\right|\right)}>\frac{10}{\sqrt{2}(3+\sqrt{2}+\sqrt{3})}>1 .
$$

For such $\alpha_{5}$ and $\rho>1, f(z)$ satisfies

$$
\left|\frac{B_{-m-\lambda} f(z)}{z^{p}}-1\right|<Q\left|a_{p+n}\right| \leq \rho\left|e^{i \beta}-\alpha_{5}\right| \quad, \quad z \in \mathbb{U} .
$$

Our next result reads as follows.

Theorem 2. If $f(z) \in A(p, n)$ satisfies

$$
\left|\left(\frac{B_{-m-\lambda+1} f(z)}{B_{-m-\lambda} f(z)}-1\right)\left(\frac{B_{-m-\lambda} f(z)}{z^{p}}-1\right)\right|<\frac{\left|e^{i \beta}-\alpha_{s}\right|^{2} n \rho^{2}}{(p+\gamma)\left(1+\left|e^{i \beta}-\alpha_{s}\right| \rho\right)} \quad, \quad z \in \mathbb{U}
$$

for some $\alpha_{s}$ defined by (13) with $\alpha_{s} \neq 1$ such that $z_{g} \in \partial \mathbb{U}(g=1,2,3, \ldots, s)$, and for some real $\rho>1$, then

$$
\left|\frac{B_{-m-\lambda} f(z)}{z^{p}}-1\right|<\rho\left|e^{i \beta}-\alpha_{s}\right| \quad, \quad z \in \mathbb{U}
$$

that is, $f(z) \in T_{p, n}\left(\alpha_{s}, \beta, \rho ; m, \lambda\right)$.

Proof. Define a function $w(z)$ by (23). Using (24) and (26), we have

$$
\left|\left(\frac{B_{-m-\lambda+1} f(z)}{B_{-m-\lambda} f(z)}-1\right)\left(\frac{B_{-m-\lambda} f(z)}{z^{p}}-1\right)\right|=\left|\frac{\left(1-e^{-i \beta} \alpha_{s}\right)^{2} z w(z) w^{\prime}(z)}{(p+\gamma)\left(1+\left(1-e^{-i \beta} \alpha_{s}\right) w(z)\right)}\right| .
$$

We suppose that there exists a point $z_{0},\left(0<\left|z_{0}\right|<1\right)$ such that

$$
\max \left\{|w(z)| ;|z| \leq\left|z_{0}\right|\right\}=\left|w\left(z_{0}\right)\right|=\rho>1 .
$$

Then, Lemma 1 leads us that $w\left(z_{0}\right)=\rho e^{i \theta},(0 \leq \theta \leq 2 \pi)$ and $z_{0} w^{\prime}\left(z_{0}\right)=k w\left(z_{0}\right),(k \geq n)$. It follows from the above that

$$
\begin{aligned}
\left|\left(\frac{B_{-m-\lambda+1} f\left(z_{0}\right)}{B_{-m-\lambda} f\left(z_{0}\right)}-1\right)\left(\frac{B_{-m-\lambda} f\left(z_{0}\right)}{z_{0}^{p}}-1\right)\right| & =\left|\frac{\left(1-e^{-i \beta} \alpha_{s}\right)^{2} z_{0} w\left(z_{0}\right) w^{\prime}\left(z_{0}\right)}{(p+\gamma)\left(1+\left(1-e^{-i \beta} \alpha_{s}\right) w\left(z_{0}\right)\right)}\right| \\
& =\frac{\left|e^{i \beta}-\alpha_{s}\right|^{2} \rho^{2} k}{(p+\gamma)\left|1+\left(1-e^{-i \beta} \alpha_{s}\right) \rho e^{i \theta}\right|} \\
& \geq \frac{\left|e^{i \beta}-\alpha_{s}\right|^{2} n \rho^{2}}{(p+\gamma)\left(1+\left|e^{i \beta}-\alpha_{s}\right| \rho\right)} .
\end{aligned}
$$

This contradicts our condition (51) for $f(z)$. Therefore, there is no $z_{0},\left(0<\left|z_{0}\right|<1\right)$ such that $\left|w\left(z_{0}\right)\right|=\rho>1$. This means that

$$
\left|\left(\frac{B_{-m-\lambda} f(z)}{z^{p}}-1\right)\right|<\rho\left|e^{i \beta}-\alpha_{s}\right|, z \in \mathbb{U}
$$


Example 2. Consider a function $f(z)$ given by (32) with $0<\left|a_{p+n}\right|<\frac{1}{Q}$, where $Q$ is given by (33). For this function $f(z)$, we have

$$
\begin{aligned}
&\left|\left(\frac{B_{-m-\lambda+1} f(z)}{B_{-m-\lambda} f(z)}-1\right)\left(\frac{B_{-m-\lambda} f(z)}{z^{p}}-1\right)\right|=\left|\frac{n Q^{2} a_{p+n}^{2} z^{2 n}}{(p+\gamma)\left(1+Q a_{p+n} z^{n}\right)}\right| \\
&<\frac{n Q^{2}\left|a_{p+n}\right|^{2}}{(p+\gamma)\left(1-Q\left|a_{p+n}\right|\right)} \quad, \quad z \in \mathbb{U} .
\end{aligned}
$$

Consider five boundary points $z_{1}, z_{2}, z_{3}, z_{4}$ and $z_{5}$ in Example 1 . Then, we have

$$
\left|1-e^{-i \beta} \alpha_{5}\right|=\frac{\sqrt{2}(3+\sqrt{2}+\sqrt{3})}{10} Q\left|a_{p+n}\right|
$$

with $\beta=0$. With such $\alpha_{5}$ and $\beta$, we take $\rho>1$ by

$$
\frac{n Q^{2}\left|a_{p+n}\right|^{2}}{(p+\gamma)\left(1-Q\left|a_{p+n}\right|\right)} \leq \frac{\left|e^{i \beta}-\alpha_{5}\right|^{2} n \rho^{2}}{(p+\gamma)\left(1+\left|e^{i \beta}-\alpha_{5}\right| \rho\right)} .
$$

Then, this $\rho$ satisfies

$$
\rho \geq \frac{10}{\sqrt{2}(3+\sqrt{2}+\sqrt{3}) Q\left|a_{p+n}\right|}>1
$$

For such $\alpha_{5}$ and $\rho$, we know that

$$
\left|\frac{B_{-m-\lambda} f(z)}{z^{p}}-1\right|<\rho\left|e^{i \beta}-\alpha_{5}\right| \quad, \quad z \in \mathbb{U} .
$$

Next, we derive the following result.

Theorem 3. If $f(z) \in A(p, n)$ satisfies

$$
\left|\frac{B_{-m-\lambda+g} f(z)}{z^{p}}-1\right|<\rho\left|e^{i \beta}-\alpha_{s}\right|\left(\frac{p+n+\gamma}{p+\gamma}\right), z \in \mathbb{U}
$$

for some $\alpha_{s}$ defined by (13) with $\alpha_{s} \neq 1, g=1,2,3, \ldots, m$, and for some real $\rho>1$, then

$$
\left|\frac{B_{-m-\lambda+g-1} f(z)}{z^{p}}-1\right|<\rho\left|e^{i \beta}-\alpha_{s}\right|, z \in \mathbb{U} .
$$

Proof. Define the function $w(z)$ by

$$
\begin{gathered}
w(z)=\frac{e^{i \beta \frac{B_{-m-\lambda+g-1} f(z)}{z^{p}}-\alpha_{s}}}{e^{i \beta}-\alpha_{s}}-1 \\
=\frac{e^{i \beta}}{e^{i \beta}-\alpha_{s}}\left\{\sum_{k=p+n}^{\infty}\left(\frac{p+\gamma}{k+\gamma}\right)^{m-g} \frac{\Gamma(k+\gamma) \Gamma(p+\gamma+\lambda)}{\Gamma(p+\gamma) \Gamma(k+\gamma+\lambda)} a_{k} z^{k-p}\right\} .
\end{gathered}
$$

It follows from the above that

$$
B_{-m-\lambda+g-1} f(z)=z^{p}+\left(1-e^{-i \beta} \alpha_{s}\right) z^{p} w(z) .
$$

By the definition of $B_{-m-\lambda+g} f(z)$, we know that

$$
B_{-m-\lambda+g} f(z)=\frac{z^{1-\gamma}}{p+\gamma}\left(z^{\gamma} B_{-m-\lambda+g-1} f(z)\right)^{\prime}=z^{p}\left\{1+\left(1-e^{-i \beta} \alpha_{s}\right) w(z)\left(1+\frac{z w^{\prime}(z)}{(p+\gamma) w(z)}\right)\right\} .
$$


Our condition implies that

$$
\left|\frac{B_{-m-\lambda+g} f(z)}{z^{p}}-1\right|=\left|\left(1-e^{-i \beta} \alpha_{s}\right) w(z)\left(1+\frac{z w^{\prime}(z)}{(p+\gamma) w(z)}\right)\right|<\rho\left|e^{i \beta}-\alpha_{s}\right|\left(\frac{p+n+\gamma}{p+\gamma}\right)
$$

for all $z \in \mathbb{U}$. Suppose that there exists a point $z_{0},\left(0<\left|z_{0}\right|<1\right)$ such that

$$
\max \left\{|w(z)| ;|z| \leq\left|z_{0}\right|\right\}=\left|w\left(z_{0}\right)\right|=\rho>1
$$

Then, Lemma 1 says that $w\left(z_{0}\right)=\rho e^{i \theta},(0 \leq \theta \leq 2 \pi)$ and $z_{0} w^{\prime}\left(z_{0}\right)=k w\left(z_{0}\right),(k \geq n)$. Therefore, we have

$$
\begin{array}{r}
\left|\frac{B_{-m-\lambda+g} f\left(z_{0}\right)}{z_{0}^{p}}-1\right|=\left|\left(1-e^{-i \beta} \alpha_{s}\right) w\left(z_{0}\right)\left(1+\frac{z_{0} w^{\prime}\left(z_{0}\right)}{(p+\gamma) w\left(z_{0}\right)}\right)\right| \\
=\rho\left|e^{i \beta}-\alpha_{s}\right|\left(1+\frac{k}{p+\gamma}\right) \geq \rho\left|e^{i \beta}-\alpha_{s}\right|\left(\frac{p+n+\gamma}{p+\gamma}\right)
\end{array}
$$

which contradicts the inequality (67). This means that there is no $z_{0} \in \mathbb{U}$ such that $\mid w\left(z_{0} \mid=\rho>1\right.$. Thus we know that

$$
|w(z)|=\left|\frac{e^{i \beta} \frac{B_{-m-\lambda+g-1} f(z)}{z^{p}}-\alpha_{s}}{e^{i \beta}-\alpha_{s}}-1\right|=\left|\frac{e^{i \beta}}{e^{i \beta}-\alpha_{s}}\left(\frac{B_{-m-\lambda+g-1} f(z)}{z^{p}}-1\right)\right|<\rho .
$$

This completes the proof of the theorem.

Theorem 3 implies the following one.

Theorem 4. If $f(z) \in A(p, n)$ satisfies

$$
\left|\frac{B_{-m-\lambda+g} f(z)}{z^{p}}-1\right|<\rho\left|e^{i \beta}-\alpha_{s}\right|\left(\frac{p+n+\gamma}{p+\gamma}\right)^{g}, z \in \mathbb{U}
$$

for some $\alpha_{s}$ given by (13) with $\alpha_{s} \neq 1, g=1,2,3, \ldots, m$, and for some real $\rho>1$, then

$$
\left|\frac{B_{-m-\lambda} f(z)}{z^{p}}-1\right|<\rho\left|e^{i \beta}-\alpha_{s}\right|, z \in \mathbb{U}
$$

or, equivalently, $f(z) \in T_{p, n}\left(\alpha_{s}, \beta, \rho ; m, \lambda\right)$.

Proof. By means of Theorem 3, we see that if $f(z)$ satisfies the inequality (71), then

$$
\left|\frac{B_{-m-\lambda+g-1} f(z)}{z^{p}}-1\right|<\rho\left|e^{i \beta}-\alpha_{s}\right|\left(\frac{p+n+\gamma}{p+\gamma}\right)^{g-1} \quad, z \in \mathbb{U}
$$

Similarly, we have

$$
\left|\frac{B_{-m-\lambda+g-2} f(z)}{z^{p}}-1\right|<\rho\left|e^{i \beta}-\alpha_{s}\right|\left(\frac{p+n+\gamma}{p+\gamma}\right)^{g-2}, z \in \mathbb{U} .
$$

Continuing this consideration, we obtain that

$$
\left|\frac{B_{-m-\lambda} f(z)}{z^{p}}-1\right|<\rho\left|e^{i \beta}-\alpha_{s}\right| \quad, \quad z \in \mathbb{U} .
$$


Example 3. Consider the function

$$
f(z)=z^{p}+a_{p+n} z^{p+n} \quad, \quad z \in \mathbb{U}
$$

which satisfies

$$
B_{-m-\lambda+g} f(z)=z^{p}+\frac{\Gamma(p+n+\gamma) \Gamma(p+\gamma+\lambda)}{\Gamma(p+\gamma) \Gamma(p+n+\gamma+\lambda)}\left(\frac{p+\gamma}{p+n+\gamma}\right)^{m-g} a_{p+n} z^{p+n}
$$

It follows from (77) that

$$
\begin{aligned}
\left|\frac{B_{-m-\lambda+g} f(z)}{z^{p}}-1\right|= & \frac{\Gamma(p+n+\gamma) \Gamma(p+\gamma+\lambda)}{\Gamma(p+\gamma) \Gamma(p+n+\gamma+\lambda)}\left(\frac{p+\gamma}{p+n+\gamma}\right)^{m-g}\left|a_{p+n}\right||z|^{n} \\
& <Q\left(\frac{p+n+\gamma}{p+\gamma}\right)^{g}\left|a_{p+n}\right| \quad, \quad z \in \mathbb{U},
\end{aligned}
$$

where $Q$ is given by (33). Now, we consider the five boundary points $z_{1}, z_{2}, z_{3}, z_{4}$ and $z_{5}$ as in Example 1 . Then we see

$$
\left|e^{i \beta}-\alpha_{5}\right|=\frac{\sqrt{2}(3+\sqrt{2}+\sqrt{3})}{10} Q\left|a_{p+n}\right|
$$

where $\beta=0$. With the above relation (79), we consider $\rho>1$ such that

$$
Q\left(\frac{p+n+\gamma}{p+\gamma}\right)^{g}\left|a_{p+n}\right| \leq \rho\left|e^{i \beta}-\alpha_{5}\right|
$$

that is, $\rho$ satisfies

$$
\rho \geq \frac{10}{\sqrt{2}(3+\sqrt{2}+\sqrt{3})}\left(\frac{p+n+\gamma}{p+\gamma}\right)^{g}>1
$$

Thus, we have that

$$
\begin{aligned}
\left|\frac{B_{-m-\lambda} f(z)}{z^{p}}-1\right| & \leq Q\left|a_{p+n}\right| \\
& \leq \rho\left|e^{i \beta}-\alpha_{5}\right|\left(\frac{p+\gamma}{p+n+\gamma}\right)^{g} \\
& <\rho\left|e^{i \beta}-\alpha_{5}\right| \quad, \quad z \in \mathbb{U} .
\end{aligned}
$$

Remark 1. If we take $\gamma=1$ in the results of this section, then these results correspond to applications of the Libera integral operator as introduced by Libera [2].

Let us write that

$$
B_{-m-\lambda} f(z)=L_{-m-\lambda} f(z)=z^{p}+\sum_{k=p+n}^{\infty}\left(\frac{p+1}{k+1}\right)^{m} \frac{\Gamma(p+1+\lambda) k !}{\Gamma(k+1+\lambda) p !} a_{k} z^{k}
$$

for $\gamma=1$ in (11). Then Theorem 1 says that if $f(z) \in A(p, n)$ satisfies

$$
\left|\frac{L_{-m-\lambda+1} f(z)}{L_{-m-\lambda} f(z)}-1\right|<\frac{\left|e^{i \beta}-\alpha_{s}\right| n \rho}{(p+1)\left(1+\left|e^{i \beta}-\alpha_{s}\right| \rho\right)} \quad, z \in \mathbb{U},
$$

for some $\alpha_{s}$ given by

$$
\alpha_{s}=\frac{1}{s} \sum_{l=1}^{s} \frac{L_{-m-\lambda} f\left(z_{l}\right)}{z_{l}^{p}}, z_{l} \in \overline{\mathbb{U}}
$$


where $-\frac{\pi}{2} \leq \beta \leq \frac{\pi}{2}$, and for some real $\rho>1$, then

$$
\left|\frac{L_{-m-\lambda} f(z)}{z^{p}}-1\right|<\left|e^{i \beta}-\alpha_{s}\right| \rho, z \in \mathbb{U} .
$$

For another result, we consider again the Libera integral operator with $\gamma=1$.

\section{Application of Carathéodory Lemma}

In this section, we will apply Carathéodory Lemma for coefficients of functions $f(z) \in A(p, n)$.

In 1907, Carathéodory [8] gave the following result.

Lemma 2. Let a function $g(z)$ given by

$$
g(z)=1+\sum_{k=1}^{\infty} c_{k} z^{k}
$$

be analytic in $\mathbb{U}$ and $\operatorname{Reg}(z)>0, z \in \mathbb{U}$. Then $g(z)$ satisfies

$$
\left|c_{k}\right| \leq 2 \quad, \quad(k=1,2,3, \ldots) .
$$

The inequality (88) is sharp for each $k$.

Applying the above lemma, we derive the following thorem.

Theorem 5. If $f(z) \in A(p, n)$ is in the class $T_{p, n}\left(\alpha_{s}, \beta, \rho ; m, \lambda\right)$, then

$$
\left|a_{k}\right| \leq \frac{2\left|e^{i \beta}-\alpha_{s}\right| \rho}{R} \quad, \quad(k=p+n, p+n+1, \ldots),
$$

where

$$
R=\left(\frac{p+\gamma}{k+\gamma}\right)^{m} \frac{\Gamma(k+\gamma) \Gamma(p+\gamma+\lambda)}{\Gamma(p+\gamma) \Gamma(k+\gamma+\lambda)} .
$$

The result is sharp for $f(z)$ given by

$$
B_{-m-\lambda} f(z)=z^{p} \frac{e^{i \theta}-\left(1+2\left|e^{i \beta}-\alpha_{s}\right| \rho\right) z}{e^{i \theta}-z} .
$$

Proof. For $f(z) \in T_{p, n}\left(\alpha_{s}, \beta, \rho ; m, \lambda\right)$, we see that

$$
\left|\frac{B_{-m-\lambda} f(z)}{z^{p}}-1\right|<\left|e^{i \beta}-\alpha_{s}\right| \rho, z \in \mathbb{U} .
$$

If we define a function $g(z)$ with $f(z) \in T_{p, n}\left(\alpha_{s}, \beta, \rho ; m, \lambda\right)$ by

$$
g(z)=\frac{\frac{B_{-m-\lambda} f(z)}{z^{p}}-\left(1-\left|e^{i \beta}-\alpha_{s}\right| \rho\right)}{\left|e^{i \beta}-\alpha_{s}\right| \rho} \quad, z \in \mathbb{U},
$$

then $g(z)$ is analytic in $\mathbb{U}$ with $g(0)=1$ and $\operatorname{Reg}(z)>0, z \in \mathbb{U}$. Also, $g(z)$ has the following power series expansion:

$$
g(z)=1+\sum_{k=p+n}^{\infty} \frac{R}{\left|e^{i \beta}-\alpha_{s}\right| \rho} a_{k} z^{k-p} .
$$


Therefore, by applying Lemma 2 to $g(z)$, we obtain

$$
\frac{R}{\left|e^{i \beta}-\alpha_{s}\right| \rho}\left|a_{k}\right| \leq 2, \quad(k=p+n, p+n+1, \ldots) .
$$

This shows the coefficient inequalities (89). Note that

$$
g(z)=\frac{e^{i \theta}+z}{e^{i \theta}-z}=1+\sum_{k=1}^{\infty} 2 e^{i \theta} z^{k}=1+\sum_{k=1}^{\infty} c_{k} z^{k}
$$

is analytic in $\mathbb{U}, g(0)=1, \operatorname{Reg}(z)>0,(z \in \mathbb{U})$ and $\left|c_{k}\right|=2,(k=1,2,3, \ldots)$. Therefore, considering $f(z)$ such that

$$
g(z)=\frac{\frac{B_{-m-\lambda} f(z)}{z^{p}}-\left(1-\left|e^{i \beta}-\alpha_{s}\right| \rho\right)}{\left|e^{i \beta}-\alpha_{s}\right| \rho}=\frac{e^{i \theta}+z}{e^{i \theta}-z},
$$

we have

$$
B_{-m-\lambda} f(z)=z^{p} \frac{e^{i \theta}-\left(1+2\left|e^{i \beta}-\alpha_{s}\right| \rho\right) z}{e^{i \theta}-z} .
$$

This completes the proof of the theorem.

Remark 2. If we take $\gamma=1$ in Theorem 5, then we get the following result for the Libera integral operator. If $f(z) \in A(p, n)$ satisfies

$$
\left|\frac{L_{-m-\lambda} f(z)}{z^{p}}-1\right|<\left|e^{i \beta}-\alpha_{s}\right| \rho, z \in \mathbb{U},
$$

then

$$
\left|a_{k}\right| \leq \frac{2\left|e^{i \beta}-\alpha_{s}\right| \rho}{R_{0}},(k=p+n, p+n+1, \ldots),
$$

where

$$
R_{0}=\left(\frac{p+1}{k+1}\right)^{m} \frac{\Gamma(p+1+\lambda) k !}{\Gamma(k+1+\lambda) p !} .
$$

The result is sharp for $f(z)$ given by

$$
L_{-m-\lambda} f(z)=z^{p} \frac{e^{i \theta}-\left(1+2\left|e^{i \beta}-\alpha_{s}\right| \rho\right) z}{e^{i \theta}-z} .
$$

Finally, we derive

Theorem 6. If $f(z) \in A(p, n)$ satisfies

$$
\sum_{k=p+n}^{\infty} R\left|a_{k}\right| \leq\left|e^{i \beta}-\alpha_{s}\right| \rho,
$$

then $f(z) \in T_{p, n}\left(\alpha_{s}, \beta, \rho ; m, \lambda\right)$, where $R$ is given by (90).

Proof. For $f(z) \in A(p, n)$, we consider

$$
\begin{aligned}
& \left|\frac{B_{-m-\lambda} f(z)}{z^{p}}-1\right|=\left|\sum_{k=p+n}^{\infty} R a_{k} z^{k}\right| \\
& <\sum_{k=p+n}^{\infty} R\left|a_{k}\right| \leq\left|e^{i \beta}-\alpha_{s}\right| \rho \quad, z \in \mathbb{U} .
\end{aligned}
$$


Therefore, if $f(z) \in A(p, n)$ satisfies (102), then we know $f(z) \in T_{p, n}\left(\alpha_{s}, \beta, \rho ; m, \lambda\right)$.

Remark 3. Letting $\gamma=1$ in Theorem 6, we have the result concerning with the Libera integral operator $L_{-m-\lambda} f(z)$.

Author Contributions: Conceptualization, S.O.; Investigation, S.O. and H.Ö.G.; Methodology, S.O.; Writing—original draft, S.O.; Writing — review and editing, H.Ö.G. All authors have read and agreed to the published version of the manuscript.

Funding: This research received no external funding.

Acknowledgments: The authors would like to give our thanks to T. Bulboaca, Department of Mathematics, Faculty of Mathematics and Computer Sciences, Babes-Bolyai University, Romania for his kind support for our paper and also the reviewers for valuable remarks and suggestions in order to revise and improve of our paper.

Conflicts of Interest: The authors declare no conflict of interest.

\section{References}

1. Bernardi, S.D. Convex and starlike univalent functions. Trans. Am. Math. Soc. 1969, 135, 429-446. [CrossRef]

2. Libera, R.J. Some classes of regular univalent functions.Proc. Am. Math. Soc. 1965, 16, 755-758. [CrossRef]

3. Owa, S. On the distortion theorems I.Kyungpook Math. J. 1978, 18, 53-59.

4. Owa, S.; Srivastava, H.M. Univalent and starlike generalized hypergeometric functions. Can. J. Math. 1987, 39, 1057-1077. [CrossRef]

5. Miller, S.S.; Mocanu, P.T. Second order differential inequalities in the complex plane.J. Math. Anal. Appl. 1978, 65, 289-305. [CrossRef]

6. Miller, S.S.; Mocanu, P.T. Differential Subordinations. In Theory and Applications; Marcel Dekker Inc.: New York, NY, USA, 2000.

7. Jack, I.S. Functions starlike and convex of order $\alpha$. J. Lond. Math. Soc. 1971, 2 , 469-474. [CrossRef]

8. Carathéodory, C. Über den Variabilitatsbereich der Fourierschen Konstanten von positiven harmonischen Funktionen. Rend. Del Circ. Mat. Palermo (1884-1940) 1911, 32, 193-217. [CrossRef]

(c) 2020 by the authors. Licensee MDPI, Basel, Switzerland. This article is an open access article distributed under the terms and conditions of the Creative Commons Attribution (CC BY) license (http:// creativecommons.org/licenses/by/4.0/). 